\title{
A complete classification of well-rounded real quadratic ideal lattices
}

\author{
Anitha Srinivasan*
}

\begin{abstract}
We provide a complete classification of well-rounded ideal lattices arising from real quadratic fields $\mathbb{Q}(\sqrt{d})$ where $d$ is a field discriminant. We show that the ideals that give rise to such lattices are precisely the ones that correspond to divisors $a$ of $d$ that satisfy $\sqrt{\frac{d}{3}}<a<\sqrt{3 d}$.
\end{abstract}

\section{Introduction}

Let $d$ be field discriminant, that is $d \equiv 1(\bmod 4)$ is square free, or $d \equiv 0$ $(\bmod 4)$ and $\frac{d}{4}$ is square free with $\frac{d}{4} \equiv 2$ or $3(\bmod 4)$. A lattice in $\mathbb{R}^{n}$ is a $\mathbb{Z}$ module generated by $n$ linearly independent vectors in $\mathbb{R}^{n}$. Recently ideal lattices have come into the forefront as a new tool for cryptography and coding theory, claiming to defeat the possible threats of a quantum attack on the hitherto established systems. Non-zero vectors in a lattice with least Euclidean norm play a key role as it is considered to be computationally difficult to find them. Therefore one is interested in a lattice that has a basis comprising of only such vectors. We call such a lattice well-rounded. Our focus will be on lattices that arise from ideals in the full ring of integers of a real quadratic field $\mathbb{Q}(\sqrt{d})$. The reader is directed to [1], [5] and [6] for more information and results on the topic. In [5] the authors exhibit infinite families of real and imaginary quadratic fields with ideals that give rise to well-rounded lattices.

*Saint Louis University- Madrid Campus, Avenida del Valle 34, 28003 Madrid, Spain. email: rsrinivasan.anitha@gmail.com 
In [6] the authors consider divisors of $d$ that satisfy $\sqrt{\frac{d}{3}}<a<\sqrt{d}$ and show that this condition is necessary and sufficient for the existence of wellrounded ideals lattices for imaginary quadratic fields $\mathbb{Q}(\sqrt{-d})$. In the case of real quadratic fields, they pose a question about whether well-rounded ideals lattices can exist when this condition is not satisfied. Our main theorem below answers this question in the affirmative. Indeed the necessary and sufficient condition given in the following theorem includes the condition given above. Note that an ideal is called well-rounded if the corresponding lattice well-rounded.

Theorem 1.1. Let $K=\mathbb{Q}(\sqrt{d})$ be a real quadratic field where $d$ is a field discriminant. A primitive ideal $I$ in the ring of integers is well-rounded if and only if $I=a \mathbb{Z}+\frac{a-\sqrt{d}}{2} \mathbb{Z}$ and $a$ is a positive integer that satisfies $\sqrt{\frac{d}{3}}<a<\sqrt{3 d}$.

Remark 1.1. The ideal $I$ in the above theorem has order dividing 2 in the class group. Indeed, $d=a^{2}-4 a c$ for some integer $c$, and hence $a=N(I)$, the norm of the ideal $I$ divides $d$. If an ideal $I$ is not primitive, then $I=(\gamma) J$, where $J$ is a primitive ideal and $\gamma$ is a positive integer. It is easy to see that $I$ is well-rounded if and only if $J$ is well-rounded. Note also that if $d=4 d_{1}$ in Theorem 1.1, then well-rounded ideals exist only if $d_{1} \equiv 3(\bmod 4)$. This is because if $d$ is even, then $a=2 a_{1}$ so that $d_{1}=a_{1}^{2}-2 a_{1} c$, from which it follows that $d_{1} \equiv 3(\bmod 4)$. This completes the answer to [6, Question 2] where the authors asked whether principal WR ideals exist in the case when $d \not \equiv 1(\bmod 4)$. We have show above that $d_{1} \equiv 2(\bmod 4)$ is not possible and [2, Proposition 4], states that there are infinitely many real quadratic fields with WR principal ideals in the case when $d_{1} \equiv 3(\bmod 4)$.

\section{Binary quadratic forms, ideals and lattices}

\section{$2.1 \quad$ Forms}

A binary quadratic form is a function $f(x, y)=a x^{2}+b x y+c y^{2}$, where $a, b, c$ are real numbers (called the coefficients of $f$ ) and $d=b^{2}-4 a c$ is the discriminant. A form is called positive definite if the discriminant is negative. We consider only integral forms, that is $a, b, c$ are integers. In the case when $\operatorname{gcd}(a, b, c)=1$, the form is primitive. Often we will suppress the variables $x$ and $y$, writing a form $f(x, y)$ as simply $f=(a, b, c)$. 
Two forms $f$ and $f^{\prime}$ are said to be equivalent, written as $f \sim f^{\prime}$, if for some $A=\left(\begin{array}{ll}\alpha & \beta \\ \gamma & \delta\end{array}\right) \in S L_{2}(\mathbb{Z})$ we have $f^{\prime}(x, y)=f(\alpha x+\beta y, \gamma x+\delta y)$. It is easy to see that $\sim$ is an equivalence relation on the set of forms of discriminant $d$.

A form $f$ is said to represent an integer $m$ if there exist coprime integers $x$ and $y$, such that $f(x, y)=m$. Note that equivalent forms represent the same integers and hence sometimes we refer to a class of forms $f$ that represents a given integer.

The set of equivalence classes of primitive forms is an abelian group called the form class group, with group law as composition given in Definition 2.1 in the next section.

If $f=(a, b, c)$, then the form $(a,-b, c)$ is the inverse of $f$. The identity form $e$ is defined as the form $\left(1,0, \frac{-d}{4}\right)$ or $\left(1,1, \frac{1-d}{4}\right)$ depending on whether $d$ is even or odd respectively. A useful fact is that any form that represents the integer 1 is equivalent to the identity form.

The infimum of a binary quadratic form $f$ is defined as $m(f)=\inf \{|f(x, y)|$ : $x, y \in \mathbb{Z}\}$, where $x, y$ are not both 0 . Note that $m(f)=m\left(f^{-1}\right)$ and $m(k f)=k m(f)$ for any real number $k$.

A form $(a, b, c)$ of negative discriminant is reduced if $|b| \leq a \leq c$ where $b>0$ in the case when $|b|=a$ or $a=c$. Symmetric forms satisfy $a=c$.

\section{$2.2 \quad$ Ideals}

Recall that $d \equiv 0,1(\bmod 4)$ denotes a field discriminant and all ideals are in the ring of integers. We present below a description of an ideal and the rule for composing two primitive ideals. The reader may refer to [3, Sections 1.1 and 1.2] for more information.

Let

$$
w=\left\{\begin{array}{lll}
\frac{1+\sqrt{d}}{2}, & d \equiv 1 & (\bmod 4) \\
\sqrt{\frac{d}{4}}, & d \equiv 0 & (\bmod 4) .
\end{array}\right.
$$

The ring of algebraic integers is the module with basis $[1, w]$. A primitive ideal $I$ can be written in the form

$$
I=a \mathbb{Z}+\frac{-b+\sqrt{d}}{2} \mathbb{Z},
$$

where $a, b$ are integers such that $a>0$ is the norm of the ideal, $0 \leq b<2 a$ and $4 a$ divides $b^{2}-d$. 
If $c=\frac{b^{2}-d}{4 a}$ then $\operatorname{gcd}(a, b, c)=1$ as $d$ is a field discriminant and so $(a, b, c)=a x^{2}+b x y+c y^{2}$ is a primitive form of discriminant $d$. Also, if $I$ is not primitive then there is a primitive ideal $J$ such that $I=(\gamma) J$ for some integer $\gamma$.

In the following definition we present the formula for the product of ideals which leads to composition of forms.

Definition 2.1. (Composition law) Let $I_{k}=a_{k} \mathbb{Z}+\frac{-b_{k}+\sqrt{d}}{2} \mathbb{Z}, \quad k=1,2$, be two primitive ideals. Let $f_{1}=\left(a_{1}, b_{1}, c_{1}\right)$ and $f_{2}=\left(a_{2}, b_{2}, c_{2}\right)$ be the corresponding binary quadratic forms of discriminant $d$. Let $g=\operatorname{gcd}\left(a_{1}, a_{2},\left(b_{1}+b_{2}\right) / 2\right)$ and let $v_{1}, v_{2}, w$ be integers such that

$$
v_{1} a_{1}+v_{2} a_{2}+w\left(b_{1}+b_{2}\right) / 2=g .
$$

If $a_{3}$ and $b_{3}$ are given by

$$
\begin{aligned}
& a_{3}=\frac{a_{1} a_{2}}{g^{2}}, \\
& b_{3}=b_{2}+2 \frac{a_{2}}{g}\left(\frac{b_{1}-b_{2}}{2} v_{2}-c_{2} w\right) \quad \bmod 2 a_{3},
\end{aligned}
$$

then $I_{1} \cdot I_{2}$ is the ideal $a_{3} \mathbb{Z}+\frac{-b_{3}+\sqrt{d}}{2} \mathbb{Z}$. Also, the composition of the forms $\left(a_{1}, b_{1}, c_{1}\right)$ and $\left(a_{2}, b_{2}, c_{2}\right)$ is the form $\left(a_{3}, b_{3}, c_{3}\right)$, where $c_{3}$ is computed using the discriminant equation.

Note that this gives the multiplication in the class group.

\subsection{Lemmas on binary quadratic forms}

The following lemma contains some elementary results on binary quadratic forms that we use in the proof of the main theorem.

\section{Lemma 2.1 .}

1. The form $(a, b, c)$ is equivalent to the form $\left(a, b+2 a \delta, a \delta^{2}+b \delta+c\right)$ for any integer $\delta$.

2. The form $(a, b, c)$ is equivalent to the form $(c,-b, a)$.

3. If $f=(a, b, c)$ is a reduced form of negative discriminant, then $a$ and $c$ are the two smallest integers represented by $f$. 
Proof. Parts are 1 and 2 are achieved by the transformation matrices $\left(\begin{array}{ll}1 & \delta \\ 0 & 1\end{array}\right)$ and $\left(\begin{array}{cc}0 & -1 \\ 1 & 0\end{array}\right)$. For the third part see [4, Chapter 6, Section 8].

In the following lemma we present an elementary fact on forms of order dividing two that is the principal tool in proving our main result.

Lemma 2.2. Let $f=(a, b, c)$ be a positive definite form of discriminant $d$ such that $f^{2} \sim e$. Then either a divides $b$ or $\frac{a^{2}}{(\operatorname{gcd}(a, b))^{2}} \geq \frac{|d|}{4}$.

Proof. Let the composition of $f$ with itself using the composition law (Definition 2.2) be $(A, B, C)$. Then $A=\left(\frac{a}{\operatorname{gcd}(a, b)}\right)^{2}$, and as equivalent forms represent the same integers, the identity form $e$ represents $A$. As $e$ is either $\left(1,0, \frac{-d}{4}\right)$ or $\left(1,1, \frac{1-d}{4}\right)$ (depending on the parity of $d$ ), from Lemma 2.1, part 3, if $A \neq 1$ then $A=\left(\frac{a}{\operatorname{gcd}(a, b)}\right)^{2} \geq \frac{|d|}{4}$. Note that $A=1$ corresponds to $a \mid b$.

\subsection{Real quadratic ideal lattices}

Let $I$ be an ideal in the ring of integers of a real quadratic field $\mathbb{Q}(\sqrt{d})$ with basis $\left[a, \frac{b-\sqrt{d}}{2}\right]$. The lattice associated to $I$ denoted by $L_{I}$ has a basis matrix

$$
A_{I}=\left(\begin{array}{cc}
a & \frac{b-\sqrt{d}}{2} \\
a & \frac{b+\sqrt{d}}{2}
\end{array}\right)
$$

so that if $\left(\begin{array}{c}m \\ n\end{array}\right) \in \mathbb{Z}^{2}$, the elements of the lattice are given by $\mathbf{x}=A_{I}\left(\begin{array}{c}m \\ n\end{array}\right)$, with norm form

$$
Q_{I}=\|\mathbf{x}\|^{2}=\left(\begin{array}{ll}
m & n
\end{array}\right) A_{I}^{T} A_{I}\left(\begin{array}{c}
m \\
n
\end{array}\right) .
$$

The lattice is well-rounded or WR if there is a basis of elements both of which have the shortest norm. We call an ideal well-rounded if the corresponding lattice is WR.

Lemma 2.3. An ideal $I$ is $W R$ if and only if its norm form $Q_{I}=t Q$, for some positive integer $t$ and $Q$ a primitive form that is equivalent to a reduced symmetric form. 
Proof. Let $I=(\gamma) J$ where $J$ is a primitive ideal. Then $A_{I}=\gamma A_{J}$ and from the norm equation (2), it follows that $Q_{I}=\gamma^{2} Q_{J}$. Let $Q$ be the primitive form obtained by dividing the three coefficients of $Q_{J}$ by their greatest common divisor $g$. Then $Q_{I}=\gamma^{2} g Q$ and the least positive integers represented satisfy $m\left(Q_{I}\right)=\gamma^{2} g m(Q)$. Clearly $I$ is WR if and only if $Q$ is WR. By Lemma 2.1, part 3, $Q$ is WR if and only if it is equivalent to a reduced symmetric form and the result follows.

\section{$3 \quad$ Proof of Theorem 1.1}

Let $I=a \mathbb{Z}+\frac{b-\sqrt{d}}{2} \mathbb{Z}$ be a primitive ideal. Recall that $d$ is a field discriminant and hence $\operatorname{gcd}(a, b, c)=1$, where $d=b^{2}-4 a c$. The corresponding lattice $L_{I}$ has norm form (given by (2))

$$
Q_{I}=2 a^{2} m^{2}+2 a b m n+\frac{b^{2}+d}{2} n^{2} .
$$

Note that $Q_{I}$ is a positive definite form of discriminant $-4 a^{2} d$.

Let $g=\operatorname{gcd}\left(2 a^{2}, 2 a b, \frac{b^{2}+d}{2}\right)$. As $d=b^{2}-4 a c$ we have $g \leq \operatorname{gcd}(a, b)$. It follows that

$$
Q=\frac{Q_{I}}{g}=\frac{2 a^{2}}{g} m^{2}+\frac{2 a b}{g} m n+\frac{b^{2}+d}{2 g} n^{2}
$$

is a primitive form of discriminant $-4 \frac{a^{2} d}{g^{2}}$.

Claim $1 Q^{2} \sim e$ if and only if $a \mid b$.

Proof. It is easy to see from Definition 2.2 that for any primitive form $(A, B, C)$, if $A \mid B$, then the composition of the form with itself gives the identity form (as $a_{3}=1$ in Definition 2.1 ). Therefore if $a \mid b$ then $Q^{2} \sim e$. Now assume that $Q^{2} \sim e$. We have $\operatorname{gcd}\left(\frac{2 a^{2}}{g}, \frac{2 a b}{g}\right)=\frac{2 a}{g} \operatorname{gcd}(a, b)$. By Lemma 2.2 either $a \mid b$ or

$$
\frac{\left(\frac{2 a^{2}}{g}\right)^{2}}{\left(\frac{2 a}{g} \operatorname{gcd}(a, b)\right)^{2}}=\frac{a^{2}}{\operatorname{gcd}(a, b)^{2}} \geq \frac{a^{2}}{g^{2}} d
$$

The above gives $d \leq \frac{g^{2}}{\operatorname{gcd}(a, b)^{2}} \leq 1(\operatorname{as~} g \leq \operatorname{gcd}(a, b))$, which is not possible. 
Claim 2 If $a \mid b$ then $Q$ is equivalent to a reduced symmetric form if and only if $a=b$ and $\sqrt{\frac{d}{3}}<a<\sqrt{3 d}$.

Proof. We first consider the case when $d \equiv 1(\bmod 4)$. As $0 \leq b<2 a$, if $a \mid b$ it follows that $b=a(\operatorname{as} b \equiv d(\bmod 4)$ is odd $)$ and $Q_{I}=\left(2 a^{2}, 2 a^{2}, \frac{a^{2}+d}{2}\right)$. Moreover $d=a^{2}-4 a c$ and $\operatorname{gcd}(a, c)=1$ gives $Q=(2 a, 2 a, a-2 c)$.

Using the equivalences given in parts 1 and 2 of Lemma 2.1, we have $Q \sim(a-2 c,-2 a, 2 a) \sim(a-2 c, 4 c, a-2 c)=f_{0}$. Observe that $f_{0}$ is symmetric. Also, $f_{0}$ is reduced if and only if $|4 c| \leq a-2 c$. Using $c=\frac{a^{2}-d}{4 a}$, it is easy to show that the condition $|4 c| \leq a-2 c$ is equivalent to $\sqrt{d}<a<\sqrt{3 d}$ when $c>0$ and to $\sqrt{\frac{d}{3}}<a<\sqrt{d}$ when $c<0$.

Now consider the case $d \equiv 0(\bmod 4)$. Let $d=4 d_{1}$ and $b=2 b_{1}$. In this case $a \mid b$ gives $b=a$ or $b=0$. We look first at the case when $a=b$. As $d_{1}=b_{1}^{2}-2 b_{1} c$ and $\operatorname{gcd}(a, b, c)=1$, it follows that $b_{1}$ and $c$ are odd. Therefore $Q_{I}=\left(2 a^{2}, 2 a b, 2\left(b_{1}^{2}+d_{1}\right)\right)=2\left(a^{2}, a b, 2 b_{1}^{2}-a c\right)=8 b_{1}\left(b_{1}, b_{1}, \frac{b_{1}-c}{2}\right)$ and thus $Q=\left(b_{1}, b_{1}, \frac{b_{1}-c}{2}\right)$. We have the equivalences (using Lemma 2.3)

$$
\begin{gathered}
\left(b_{1}, b_{1}, \frac{b_{1}-c}{2}\right) \sim\left(\frac{b_{1}-c}{2},-b_{1}, b_{1}\right) \sim\left(\frac{b_{1}-c}{2},-b_{1}+2 \frac{b_{1}-c}{2}, \frac{b_{1}-c}{2}\right) \\
\sim\left(\frac{b_{1}-c}{2}, c, \frac{b_{1}-c}{2}\right)=f_{0} .
\end{gathered}
$$

The form $f_{0}$ is reduced iff $|c| \leq \frac{b_{1}-c}{2}$. In an identical fashion to the case above, noting that $a=b=2 b_{1}$ we obtain that $f_{0}$ is reduced if and only if $\frac{\sqrt{d}}{3}<a<\sqrt{3 d}$. To complete the proof of Claim 2 it remains to consider the case $b=0$. We have $d_{1}=-a c$ and hence $Q_{I}=\left(2 a^{2}, 0,-2 a c\right)$ and $Q=(a, 0,-c)$. One of $(a, 0,-c)$ or $(-c, 0, a)$ is reduced and clearly neither is symmetric as $a \neq-c(\operatorname{gcd}(a, c)=1)$.

From Lemma 2.3 the ideal $I$ is $\mathrm{WR}$ if and only if $Q$ is equivalent to a reduced symmetric form. From Claims 1 and 2 it follows that $I$ is WR if and only if $a=b$ and $\sqrt{\frac{d}{3}}<a<\sqrt{3 d}$.

\section{References}

[1] M.T. Damir, D Karpuk, Well-rounded twists of ideal lattices from real quadratic fields, Journal of Number Theory, 196 (March 2019), 168-196. 
[2] O.W. Gnilke, H.T.N Tran, A. Karrila, C. Hollanti, Well-Rounded Lattices for Reliability and Security in Rayleigh Fading SISO Channels, https://arxiv.org/pdf/1605.00419.pdf

[3] R. A. Mollin, Quadratics. CRC press, Boca Raton, 1996.

[4] P. Ribenboim, My Numbers, My Friends, Popular Lectures on Number Theory. Springer-Verlag, 2000.

[5] L. Fukshansky and K. Peterson On well-rounded ideal lattices, Int. J. of Number Theory 8 (1) (2012) 189-206.

[6] L. Fukshansky, G. Henshaw, P. Lao, M. Prince, X. Sun, S. Whitehead, On well-rounded ideal lattices - II, Int. J. of Number Theory 9 (1) (2013) 139-154. 